\title{
A web-based intervention (RESTORE) to support self-management of cancer-related fatigue following primary cancer treatment: a multi-centre proof of concept randomised controlled trial
}

\author{
Claire Foster $^{1} \cdot$ Chloe Grimmett $^{1} \cdot$ Christine M. May $^{1} \cdot$ Sean Ewings $^{2} \cdot$ Michelle Myall $^{1}$. \\ Claire Hulme $^{3}$ - Peter W. Smith ${ }^{2}$ - Cassandra Powers ${ }^{1} \cdot$ Lynn Calman $^{1} \cdot$ Jo Armes $^{4}$. \\ Matthew Breckons ${ }^{5}$ • Jessica Corner ${ }^{6}$ Deborah Fenlon ${ }^{6}$ - Lynn Batehup ${ }^{6}$ • \\ Elaine Lennan ${ }^{7} \cdot$ Carl R. May $^{6} \cdot$ Carolyn Morris $^{8} \cdot$ Amanda Neylon $^{9} \cdot$ Emma Ream $^{10}$. \\ Lesley Turner $^{8}$ - Lucy Yardley ${ }^{11}$ - Alison Richardson ${ }^{6,7}$
}

Received: 18 August 2015 / Accepted: 23 November 2015 /Published online: 7 December 2015

(C) The Author(s) 2015. This article is published with open access at Springerlink.com

\begin{abstract}
Purpose Cancer-related fatigue (CRF) is a frequent and distressing symptom experienced after cancer treatment. RESTORE is the first web-based resource designed to enhance self-efficacy to manage CRF following curative-intent treatment. The aim of this study is to test the proof of concept and inform the design of an effectiveness trial.

Methods A multi-centre parallel-group two-armed (1:1) exploratory randomised controlled trial (RCT) with qualitative process evaluation was employed in the study. Participants ( $\geq 18$ years; $\leq 5$ years post treatment with moderate to severe fatigue) were recruited and randomly assigned to RESTORE or a leaflet. Feasibility and acceptability were measured by recruitment, attrition, intervention adherence, completion of outcome measures and process evaluation. Change in self-
\end{abstract}

efficacy to manage CRF was also explored. Outcome measures were completed at baseline (T0), 6 weeks (T1) and 12 weeks (T2). Data were analysed using mixed-effects linear regression and directed content analysis.

Results One hundred and sixty-three people participated in the trial and 19 in the process evaluation. The intervention was feasible (39\% of eligible patients consented) and acceptable (attrition rate $36 \%$ ). There was evidence of higher fatigue self-efficacy at $\mathrm{T} 1$ in the intervention group vs comparator (mean difference 0.51 [ -0.08 to 1.11$]$ ), though the difference in groups decreased by 12 weeks. Time since diagnosis influenced perceived usefulness of the intervention. Modifications were suggested.

Conclusion Proof of concept was achieved. The RESTORE intervention should be subject to a definitive trial with some
Claire Foster

C.L.Foster@soton.ac.uk

1 Macmillan Survivorship Research Group, Faculty of Health Sciences, University of Southampton, Highfield, Southampton SO17 1BJ, UK

2 Southampton Statistical Research Institute, University of Southampton, Highfield, Southampton SO17 1BJ, UK

3 Academic Unit of Health Economics, Leeds Institute of Health Sciences, University of Leeds, Leeds LS2 9LJ, UK

4 Florence Nightingale School of Nursing \& Midwifery, King's College, London SE1 8WA, UK
Institute of Health \& Society, Newcastle University, Newcastle upon Tyne NE2 4AX, UK

6 Faculty of Health Sciences, University of Southampton, Highfield, Southampton SO17 1BJ, UK

7 University Hospital Southampton NHS Foundation Trust, Southampton General Hospital, Southampton SO16 6YD, UK

8 Research Partner, London, UK

9 Macmillan Cancer Support, London SE1 7UQ, UK

10 Faculty of Health and Medical Sciences, University of Surrey, Guildford, Surrey GU2 7JP, UK

11 Psychology, University of Southampton, Highfield, Southampton SO17 1BJ, UK 
adjustments. Provision of an effective supportive resource would empower cancer survivors to manage CRF after treatment completion.

\section{Trial registration ISRCTN67521059}

Keywords Cancer $\cdot$ Fatigue $\cdot$ Web-based

Self-management $\cdot$ Intervention

\section{Background}

With improvements in cancer detection and treatment, the number of cancer survivors is steadily rising [1]. However, the consequences of cancer and its treatment can have a significant impact on health services and individuals' daily lives [2]. There is growing demand for tailored resources to support survivors to live with the consequences of cancer, including supported self-management, to address individual needs [3].

Cancer-related fatigue (CRF) is a frequently reported and distressing symptom [4], is associated with depression [5] and can persist for years [6] after treatment completion. CRF can be difficult to manage; pharmacological solutions have limited effect and there has been a call for non-pharmacological interventions [7]. Physical activity [8], cognitive behaviour therapy and psychosocial support [9] may improve CRF, but existing programmes are not accessible to many and often require significant input from trained professionals to deliver them.

Evidence is growing that Internet-based interventions can help people manage physical symptoms [10]. The Internet can reach a large population in a cost-effective way: $87 \%$ of the British and $74 \%$ of the US populations have access to the Internet with the over 50s being the fastest growing group [11, 12]. Furthermore, with changes in cancer aftercare, particularly a move towards supported self-management, resources to support patients to self-manage are of utmost importance.

Foster and Fenlon [13] devised a conceptual framework of recovery of health and wellbeing following cancer treatment which assumes that cancer and its treatment disrupt people's lives, and requires a process of recovery. The framework is underpinned by principles of self-efficacy theory [14]. Belief in one's ability to manage consequences of cancer and its treatment may be low after treatment [15], and support to enhance self-efficacy to live with problems, and thus improve health and wellbeing, is likely to be an important part of recovery. Few web-based interventions for cancer survivors consider CRF [16, 17], and none have explicitly attempted to improve self-efficacy to self-manage CRF.

Interventions designed to increase self-efficacy to manage physical symptoms have been shown to be effective in chronic-disease populations, and several self-management programmes are designed to enhance self-efficacy [18]. Increased self-efficacy to manage a symptom may alter a person's perception of that symptom, making it feel less bothersome to everyday life, thus enhancing quality of life (QoL) [19].

The aim of this exploratory randomised controlled trial (RCT) and process evaluation was to test the proof of concept of RESTORE, a web-based resource designed to increase selfefficacy to manage CRF. Proof of concept was tested by exploring feasibility, acceptability and potential to increase selfefficacy to self-manage CRF following primary cancer treatment. Data from this study will also inform a sample size calculation for a full-scale effectiveness RCT. Participants were introduced to the trial in secondary care to test the feasibility of embedding the resource in this context. This paper presents findings of the exploratory RCT, following Consolidated Standards of Reporting Trials (CONSORT) guidelines [20] and informed by Standard Protocol Items: Recommendations for Interventional Trials (SPIRIT) guidance for clinical trials [21]. Impact on health care resource use will also be explored.

\section{Methods}

\section{The intervention}

RESTORE was developed using LifeGuide open-source software [22]. Full details of the development of RESTORE are provided elsewhere [23].

RESTORE consisted of five sessions informed by Macmillan Cancer Backup's leaflet, Coping with Fatigue [24]; self-efficacy theory; cognitive behavioural therapy; and evidence of fatigue management in cancer survivors. The content of RESTORE is described elsewhere [25]. Briefly, participants were limited to 6 weeks access to RESTORE and presented with sessions at weekly intervals. Sessions 1 and 2 were mandatory and introduced CRF and goal setting. For the next 3 weeks, participants chose from (i) diet, sleep, exercise, home and work life; (ii) thoughts and feelings; and (iii) talking to others. Participants can choose to complete all available sessions or spend more time on the area/s most important to them. Structured activities were available throughout including goal setting, automated tailored feedback on achievement of goals and fatigue level, and videos of patient stories. Participants were also encouraged to download and complete a fatigue diary.

\section{Design}

A parallel-group two-armed (1:1) exploratory RCT with a qualitative process evaluation was employed. Participants were randomised to RESTORE or the leaflet. 


\section{Participants}

Eligible participants were $\geq 18$ years, had clinical diagnosis of invasive cancer $\leq 5$ years previously, had completed curativeintent treatment (surgery/cytotoxic chemotherapy/radiotherapy), had no evidence of metastatic disease, self-reported moderate to severe fatigue (fatigue of $\geq 4$ on an 11-point rating scale) [26] and had access to the Internet and an email account or willing to create an email account. Individuals were excluded if their clinical care team deemed them unable to give informed consent, they had a mental health condition likely to be exacerbated by participation or they were too ill to engage in the study.

\section{Procedures}

Approvals were granted by the National Research Ethics Service Committee (REC: 12/SC/0374) and University Hospital Southampton NHS Foundation Trust (REF RHM CAN0875).

Participants were recruited between September 2012 and May 2013 from 12 sites across the UK. Medical details were collected for all consented participants. Reasons for nonparticipation were recorded wherever possible.

A subsample of participants were invited by email to take part in the process evaluation. A maximum-variation sample was drawn across trial arms, participating sites and a range of individual characteristics (gender, age, adherence with RESTORE).

\section{Data collection}

During screening, date of birth, sex and cancer type were recorded. Participants provided informed consent and completed baseline (T0) questionnaires online. Information from clinical records included cancer diagnosis (date and type) and treatments received.

The RESTORE group received automated weekly emails announcing the availability of their next session and reminders if they had not accessed the session within 7 days. Participants completed further questionnaires at 6 (T1) and 12 weeks (T2) post baseline. RESTORE was available to both groups after completion of T2 questionnaires.

Process evaluation interviews, conducted by telephone after T2 assessments, were recorded and transcribed verbatim. Pseudonyms replaced names.

\section{Measures}

The following validated measures were assessed at each time point:

- Perceived Self-efficacy for Fatigue Self-management (PSEFSM) includes 6 items assessing degree of self- efficacy in performing various tasks on an 11-point scale (score 0-10; higher scores $=$ greater self-efficacy). This measure has good reliability (Cronbach's coefficient of 0.92 ), construct validity and generalisability [27].

- Cancer Survivors' Self-efficacy Scale (CS-SES) [28] is an 11-item scale, based on Lorig's 6-item Chronic Disease Self-efficacy Scale [29]. Participants rate their confidence in performing various disease-related activities (scale of 0-10; higher score $=$ higher self-efficacy).

- Functional Assessment of Cancer Therapy-General (FACT-G) [30] is a 27-item questionnaire consisting of 4 subscales: physical, social, emotional and functional wellbeing (total score of 0-108; higher scores=better QoL).

- Personal Wellbeing Index (PWI) [31] includes 7 items related to 'How satisfied are you with your life as a whole?' Response options are 0 to 10 (higher score= higher satisfaction with life; scale 0-100).

- Patient Health Questionnaire (PHQ-9) [32] is a 9-item scale based on the diagnostic criteria for depressive disorder measuring symptoms/functional impairment and severity (total score range $0-27$; higher score=greater depression).

- Brief Fatigue Inventory (BFI) [33] is a 9-item scale assessing current, usual and worst levels of fatigue (in the past $24 \mathrm{~h}$ ) and the extent to which fatigue interferes with everyday life (scale of $0-10$; higher scores $=$ more fatigue). A cut-off of $\geq 7$ denotes 'severe' fatigue [33].

Intervention adherence was examined by usage data. Accessing session 3 was chosen as the cut-off for adherence as this is where participants begin to set goals and engage with other activities not available in the leaflet. Data on health service use was collected by bespoke patient questionnaire. Finally, process evaluation interviews explored feasibility and acceptability of the trial [34], e.g. the perceived usefulness of RESTORE/leaflet, the work required to complete the study and suggested improvements to RESTORE.

\section{Sample size}

A formal sample size calculation was inappropriate for this exploratory RCT; a sample of 125 was chosen pragmatically, based on resources and time available. Estimated attrition was $20 \%$.

\section{Randomisation}

A statistician independently generated a random allocation sequence, using ' $\mathrm{R}$ ' for each NHS centre, and participants were randomised in blocks of four [20]. Participants were randomised by the research team after completion of $\mathrm{T} 0$ 
questionnaires. Statisticians and members of the research team not involved in recruitment were blinded during analysis.

\section{Analysis}

Feasibility of RESTORE was assessed by recruitment rates, reasons for non-participation and attrition. Acceptability was inferred by adherence with the intervention and questionnaire completion rates. Feasibility and acceptability were further explored in the process evaluation. Transcripts were analysed using directed content analysis within a framework approach.

The potential for RESTORE to improve self-efficacy was examined by a mixed-effects model for fatigue self-efficacy with a random intercept for centre (to account for withincentre correlation) and random coefficients for time (to account for within-person correlation). The effect of group (comparator vs intervention) in this model was of primary interest. Analyses were based on intention-to-treat.

Health economic analysis identified health care resource use across groups and is reported using descriptive statistics.

\section{Results}

\section{Participants}

Figure 1 shows patient flow through the study. Three participants consented but did not complete a T0 questionnaire (not randomised). Recruitment was faster than expected and 163 participants were randomised, 85 allocated to RESTORE and 78 the leaflet. (See Table 1 for sample characteristics.) Four participants experienced a recurrence during the study and were excluded from analysis.

Nineteen of the 81 invited participants completed a process evaluation interview, 8 from RESTORE and 11 from the comparator group. Most $(n=15)$ were female and $\leq 60$ years $(n=$ 14 , age range 39-78 years). A range of cancer types were represented; the majority $(n=12)$ had breast cancer. Six in the RESTORE group had accessed $\geq 3$ RESTORE sessions.

\section{Feasibility}

One hundred and sixty-three participants were recruited, an average of five participants per week. Forty-one percent of eligible participants consented to the study ( $16 \%$ of those screened). Nine percent of patients screened were ineligible due to a lack of access to a computer/Internet, and a further $1 \%$ declined due to the web-based nature of the study.

Randomisation worked well; baseline characteristics were generally well-balanced between groups (Table 1). However, the intervention group had a higher proportion 'not working' (48.8 vs $36.0 \%$ ), primarily due to more retired people in this group, and a greater number of days since last cytotoxic treatment (577.71 vs 484.61).

No process evaluation participants reported having to learn new skills to use RESTORE. However, concerns were raised that older people might struggle if they did not use computers regularly. Some participants encountered problems navigating RESTORE, experienced difficulties logging on, had password refused and reported screens freezing or closing down unexpectedly.

\section{Acceptability}

Half of participants in the process evaluation felt the timing of participation was 'about right'. The remainder would have preferred RESTORE sooner. They were $\geq 1$ year from diagnosis and suggested benefits of participating before treatment completion.

Most participants identified benefits of taking part in the trial, including feeling supported and reassured that someone was interested in their condition. A number made positive changes to their lifestyle as a result of using RESTORE or the leaflet. For some, RESTORE had improved their understanding of CRF and how to manage it; for others, involvement in the trial offered a period of reflection and allowed them to re-evaluate what they could do. Most reported an increase in confidence to self-manage the effects of CRF and considered their CRF to be less bothersome.

Of those who accessed both RESTORE and the leaflet, half preferred RESTORE, finding it more flexible and interactive. Others felt the leaflet was more convenient as it was immediately available and could be consulted any time.

Suggested improvements to the intervention included providing more cancer-specific information and more personalised feedback.

Total attrition rate (consent to T2) was $36 \%$. Nine people (5.5\%) actively withdrew and $36(22.1 \%)$ did not complete follow-up questionnaires. Seven participants from the RESTORE group, and 2 from the comparator group missed $\mathrm{T} 1$ but completed T2 questionnaires. Four people were excluded due to metastatic disease. No adverse events were reported.

Seventy-one percent of participants were deemed to have adhered with the intervention (logged on to sessions 1 and 2 and a third session). Sixty percent logged on to four sessions, $43 \%$ to five sessions. The Work and home life session was the most visited (51\% of participants) of the three 'optional' sessions, and Talking to others was the least visited ( $27 \%$ of participants). Within sessions 3-5, participants engaged most with the goal-setting section and least with the goal review section.

\section{Potential to increase self-efficacy to manage CRF}

Missing data were handled using multiple imputation where appropriate. Ninety-seven $(61.0 \%)$ complete cases were 


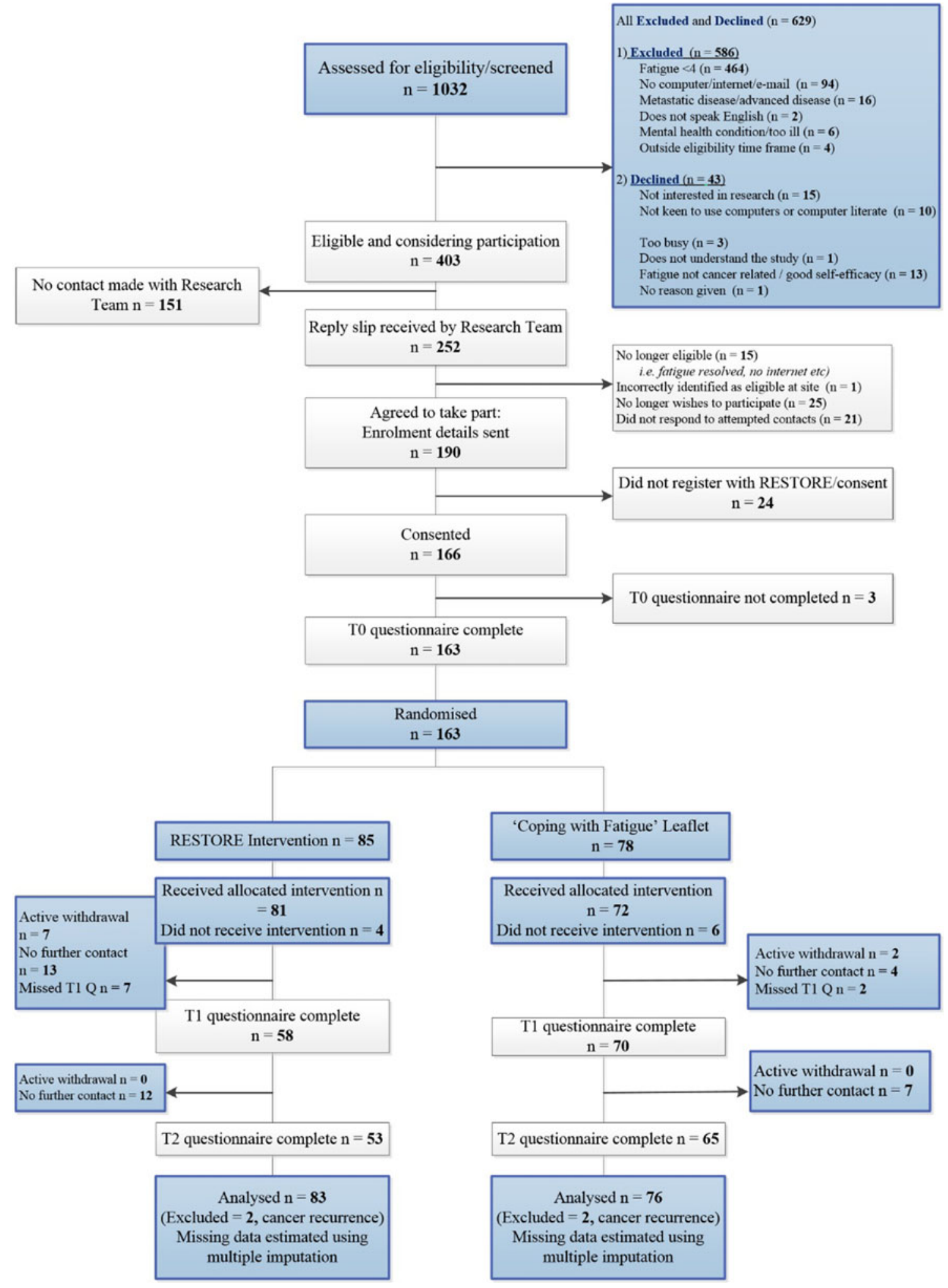

Fig. 1 Patient flow

observed and 130 (81.8\%) people had $\leq 6$ missing values. Imputation models were constructed for all outcomes at each time point. As a typical example, the imputation model for fatigue self-efficacy at $\mathrm{T} 1$ included fatigue self-efficacy at baseline and $\mathrm{T} 2$, each of the other outcomes at $\mathrm{T} 1$, centre, group, age, gender and time since last treatment. Examination of partial correlations between all outcomes suggested the inclusion of baseline values of fatigue self-efficacy 
Table 1 Baseline characteristics of RESTORE and comparator groups

\begin{tabular}{|c|c|c|c|c|}
\hline & & All $(n=159)$ & $\begin{array}{l}\text { RESTORE } \\
(n=83)\end{array}$ & $\begin{array}{l}\text { Comparator } \\
(n=76)\end{array}$ \\
\hline \multirow[t]{2}{*}{ Gender, $n(\%)$} & Male & $37(23.3)$ & $22(26.5)$ & $15(19.7)$ \\
\hline & Female & $122(76.7)$ & $61(73.5)$ & $61(80.3)$ \\
\hline \multirow[t]{2}{*}{ Age } & Range & $29-80$ & $29-78$ & $35-80$ \\
\hline & Mean (SD) & $57.8(9.95)$ & $58.1(10.7)$ & $57.5(9.1)$ \\
\hline \multirow[t]{2}{*}{ Ethnicity, $n(\%)$} & White & $156(98.7)$ & $81(97.6)$ & $75(100)$ \\
\hline & Non-white & $2(1.3)$ & $2(2.4)$ & $0(0)$ \\
\hline \multirow{5}{*}{$\begin{array}{l}\text { IMD quintile (England only; } \\
\qquad n=140), n(\%)\end{array}$} & 1 (most deprived) & $16(11.4)$ & $7(9.5)$ & $9(13.6)$ \\
\hline & 2 & $23(16.4)$ & $13(17.6)$ & $10(15.2)$ \\
\hline & 3 & $34(24.3)$ & $19(25.7)$ & $15(22.7)$ \\
\hline & 4 & $34(24.3)$ & $18(24.3)$ & $16(24.2)$ \\
\hline & 5 & $33(23.6)$ & $17(23.0)$ & $16(24.2)$ \\
\hline \multirow[t]{2}{*}{ Employment status, $n(\%)$} & Employed & $90(57.8)$ & $42(51.2)$ & $48(64.0)$ \\
\hline & Unemployed & $67(42.2)$ & $40(48.8)$ & $27(36.0)$ \\
\hline \multirow{4}{*}{$\begin{array}{l}\text { Other long-term conditions, e.g. } \\
\quad \text { diabetes, asthma; } n(\%)\end{array}$} & 0 & $54(34.0)$ & $28(33.7)$ & $26(34.2)$ \\
\hline & 1 & $54(34.0)$ & $27(32.5)$ & $27(35.5)$ \\
\hline & 2 & $29(18.2)$ & $18(21.7)$ & $11(14.5)$ \\
\hline & $>2$ & $22(13.8)$ & $10(12.0)$ & $12(15.8)$ \\
\hline \multirow[t]{7}{*}{ Cancer type, $n(\%)$} & Breast & $94(59.1)$ & $46(55.4)$ & $48(63.2)$ \\
\hline & GI & $25(15.7)$ & $14(16.9)$ & $11(14.5)$ \\
\hline & Bladder/kidney & $1(0.6)$ & $0(0)$ & $1(1.3)$ \\
\hline & Gynaecological & $8(5.0)$ & $5(6.0)$ & $3(3.9)$ \\
\hline & Head and neck & $15(9.4)$ & $10(12.0)$ & $5(6.6)$ \\
\hline & Lung & $2(1.3)$ & $0(0)$ & $2(2.6)$ \\
\hline & Prostate & $14(8.8)$ & $8(9.6)$ & $6(7.9)$ \\
\hline \multirow[t]{4}{*}{ Treatment type, $n(\%)$} & Chemotherapy & $110(69.2)$ & $52(62.7)$ & $58(76.3)$ \\
\hline & Radiotherapy & 109 (68.6) & $53(63.9)$ & $56(73.7)$ \\
\hline & Surgery & $135(84.9)$ & $71(85.5)$ & $64(84.2)$ \\
\hline & Hormone/endocrine & $74(46.5)$ & $37(44.6)$ & $37(48.7)$ \\
\hline Time since diagnosis (days) & & $771(569)$ & $768(584)$ & $773(557)$ \\
\hline $\begin{array}{l}\text { Time since last cytotoxic } \\
\text { treatment (days) }\end{array}$ & & $531(524)$ & $578(622)$ & $485(405)$ \\
\hline
\end{tabular}

$I M D$ index of multiple deprivation and BFI in each imputation model. Time since last treatment also required multiple imputation (missing $n=15$ [9.4\%]); the imputation model included all baseline values of outcomes, centre, group, age and gender.

Multiple imputation was performed using Stata 12.1. Predictive mean matching was used to ensure feasible values of the outcomes, with imputations drawn from the nearest three neighbours. Inspection of histograms suggested observed and imputed values had similar distributions.

The intended analysis (between-group, repeated-measures model with random intercept for centre and random coefficients for time) could not be implemented due to failure of the model to converge; we believe the highly variable nature of how fatigue evolves over time in an individual is the reason for this. Instead, simpler mixed-effects models were used to assess the effect of the intervention on outcomes at T1 and T2 separately (controlling for baseline scores and with a random intercept for centre).

Table 2 shows mean scores for all outcome measures over the three time points. There is evidence of improved fatigue self-efficacy at T1 $(0.514,95 \% \mathrm{CI}[-0.084,1.112], P=0.09)$, in the RESTORE group though the impact is lost by T2. There is no evidence of difference between groups for any other outcomes.

The pattern of fatigue self-efficacy for adherent and nonadherent individuals was similar to the patterns observed for the RESTORE and comparator groups: T0 5.466 (1.944) vs $5.028(1.827)$ and T1 $6.520(1.789)$ vs 5.250 (1.250).

Based on data observed, a sample of at least 317 per group would be required to detect a standardised effect size of 0.5 (significance level $5 \%$ and power of $90 \%$ ). 
Table 2 Means, standard deviation and estimated group effects for fatigue self-efficacy and all secondary outcome measures

\begin{tabular}{|c|c|c|c|c|c|}
\hline & \multirow[t]{2}{*}{ Time point } & \multicolumn{2}{|l|}{ Mean (SD) } & \multirow[t]{2}{*}{ Group effect $(95 \% \mathrm{CI})$} & \multirow[t]{2}{*}{$P$} \\
\hline & & RESTORE & Comparator & & \\
\hline \multirow[t]{3}{*}{ Fatigue self-efficacy (range $1-11)^{\mathrm{a}}$} & $\mathrm{T} 0$ & $5.376(1.930)$ & $5.373(2.048)$ & - & - \\
\hline & $\mathrm{T} 1$ & $6.421(1.781)$ & $5.904(2.107)$ & $0.514(-0.084,1.112)$ & 0.09 \\
\hline & $\mathrm{T} 2$ & $6.439(2.228)$ & $6.294(2.207)$ & $0.106(-0.427,0.638)$ & 0.70 \\
\hline \multirow[t]{3}{*}{ Cancer survivor self-efficacy (range $0-10)^{\mathrm{a}}$} & $\mathrm{T} 0$ & $6.79(1.58)$ & $6.68(1.75)$ & - & - \\
\hline & $\mathrm{T} 1$ & $7.13(1.57)$ & $7.05(1.63)$ & $0.026(-0.359,0.411)$ & 0.90 \\
\hline & $\mathrm{T} 2$ & $7.35(1.79)$ & $7.23(1.77)$ & $0.160(-0.235,0.556)$ & 0.43 \\
\hline \multirow[t]{3}{*}{ Brief Fatigue Inventory (range $0-10$ ) } & $\mathrm{T} 0$ & $5.41(1.86)$ & $5.41(1.96)$ & - & - \\
\hline & $\mathrm{T} 1$ & $5.08(2.39)$ & $4.62(2.17)$ & $0.353(-0.293,0.999)$ & 0.28 \\
\hline & $\mathrm{T} 2$ & $4.34(2.50)$ & $4.36(2.36)$ & $-0.239(-0.938,0.459)$ & 0.50 \\
\hline \multirow[t]{3}{*}{ Personal Wellbeing Index (range $0-100)^{\mathrm{a}}$} & $\mathrm{T} 0$ & $64.9(17.2)$ & $63.0(19.8)$ & - & - \\
\hline & $\mathrm{T} 1$ & $65.3(19.1)$ & $64.6(18.6)$ & $0.622(-3.437,4.682)$ & 0.76 \\
\hline & $\mathrm{T} 2$ & $63.8(21.8)$ & $65.1(24.1)$ & $0.244(-5.687,6.175)$ & 0.94 \\
\hline \multirow[t]{3}{*}{ FACT-G (range $0-108)^{a}$} & $\mathrm{~T} 0$ & $72.9(16.2)$ & $71.4(17.8)$ & - & - \\
\hline & $\mathrm{T} 1$ & $74.1(18.0)$ & $76.9(17.4)$ & $-2.206(-5.503,1.091)$ & 0.19 \\
\hline & $\mathrm{T} 2$ & $75.0(19.4)$ & $78.7(18.5)$ & $-3.034(-6.639,0.571)$ & 0.10 \\
\hline \multirow[t]{3}{*}{ PHQ-9 (range 0-27) } & T0 & $9.77(5.50)$ & $8.96(5.66)$ & - & - \\
\hline & $\mathrm{T} 1$ & $8.41(5.58)$ & $7.74(5.82)$ & $-0.452(-1.761,0.858)$ & 0.50 \\
\hline & $\mathrm{T} 2$ & $8.59(6.37)$ & $6.82(5.50)$ & $0.676(-0.880,2.231)$ & 0.40 \\
\hline
\end{tabular}

\section{Health resource use}

There was a high proportion of missing health economic data, making an economic evaluation challenging and formal statistical comparisons impossible. Examination of available descriptive data suggests comparable use of resources between groups: mean number of visits to a GP practice at T2 2.29 (1.27) in the RESTORE and 1.90 (1.04) in the comparator groups, and $1.41(0.80)$ and $1.29(1.27)$ visits to the oncologist respectively.

\section{Discussion}

The primary aim of the study was to test proof of concept by establishing the feasibility and acceptability of RESTORE and the potential to improve fatigue self-efficacy following treatment with curative intent. The intervention was found to be feasible and acceptable and established proof of concept. Uptake of the intervention was high (39\%) compared to that of similar interventions [35], suggesting a demand for supportive resources in this population. Although not powered to detect change, there was evidence of higher fatigue selfefficacy at T1 in the RESTORE group compared with the comparator group, though improvements in the comparator group meant the difference between groups was negligible by 12 weeks.
Participants in the process evaluation did not have to learn new skills to take part but felt those less familiar with the Internet would need support. Dropout was higher in the RESTORE arm and this may be because they struggled with the web-based nature of the trial.

Despite being a problem that is widely reported during treatment and in the year following treatment [7, 36], fatigue is not routinely assessed or discussed. In this study, many people were experiencing fatigue in the years following treatment and found participation in the trial beneficial as an acknowledgement of their fatigue and its impact. This complements results from Yun et al. [16] who describe the only other web-based intervention for CRF, reporting improvements in CRF following a 12-week education programme.

There appears to be no discernible pattern to fatigue selfefficacy over time in this sample. This may in part be due to the complex nature of CRF and the wide range of time since treatment completion ( $\leq 5$ years). For some, fatigue may be relatively short-lived; for others, it may have persisted for several years. Those with long-standing fatigue are likely to have tried a number of different strategies to manage their CRF. Other studies have identified the process of trial and error in relation to self-management of chronic conditions [37]. Therefore, timing of an intervention like RESTORE is likely to be highly important. Our results suggest that offering RESTORE soon after treatment completion may have the greatest benefit. 
More people are turning to the Internet for health-related information but the need to reduce digital exclusion is recognised [38]. Only $9 \%$ of those screened for this study were deemed ineligible due to a lack of computer or Internet access; nevertheless, our results suggest some individuals have a preference for written media. Individual preferences are likely to be important for the success of resources such as RESTORE and need to be considered in future work-e.g. asking people if they would prefer web-based, leaflet or a combination.

RESTORE appeared to have additional benefits when compared to the leaflet through the accessibility of the resource and the structured activities. There was evidence that participants engaged with RESTORE and gained benefit from doing so, in particular the goal-setting sections and interactive nature of the intervention. These components have been shown to be effective in other studies [39]. Some participants were more adherent than others, and these individuals appear to have yielded more benefit although a larger trial will enable us to test this.

The proportion of missing data across outcome measures was consistent, suggesting no single measure is particularly (un)suitable as a primary outcome in a future study, though methods to reduce missing data rates will be required. Low completion rates of economic data suggest these data collection methods should be simplified or condensed through, for example, use of alternative sources of routinely collected data such as Hospital Episode Statistics.

Participant dropout was higher than expected (particularly in the RESTORE group) and higher than reported in comparable studies $[16,17]$. This was explored in the process evaluation where some areas of improvement were identified, e.g. timing and choice of paper-based and web-based content. Measures to improve engagement with the resource need to be further explored. Higher dropout rates are not unusual for web-based intervention studies [40]. Reasons cited for this include the severity and chronic nature of the condition [41], loss of interest and salient content. However, there is a paucity of data on approaches to sustain usage, and some data suggest participants who drop out may still benefit from the intervention [42].

\section{Limitations}

This study has a number of limitations. We compared RESTORE against a leaflet which was also an intervention. Our rationale for doing this was the importance of determining if RESTORE is likely to yield greater benefits than an existing resource. However, we were unable to restrict independent access to a publicly available resource by the RESTORE group. In addition, it was not possible to blind participants to group allocation. Additionally, we worked closely with recruiting sites through study days to talk through study processes and procedures. We involved people affected by CRF and they gave powerful accounts of their experiences of CRF. This raised awareness of CRF among research nurses who had not considered this to be a significant problem, and consequently, there were several reports of changes in practice. Process evaluation results should be interpreted with caution due to the low response rate. Finally, external generalisability is affected by the larger proportion of women (and participants with breast cancer) in the study.

The findings from this exploratory trial suggest that RESTORE is feasible and acceptable and warrants testing in a larger efficacy trial. However, a number of refinements to RESTORE are required before testing its effectiveness in a large trial, for example including cancer-site-specific information, providing more personalised feedback on progress and targeting the intervention for participants within 12 months of treatment completion. This is a potentially important form of support for the growing numbers of cancer survivors living with and managing consequences of cancer and its treatment.

Abbreviations: BFI Brief Fatigue Inventory, CONSORT Consolidated Standards of Reporting Trials, CRF Cancerrelated fatigue, CS-SES Cancer Survivors' Self-efficacy Scale, FACT-G Functional Assessment of Cancer Therapy - General, IMD Index of Multiple Deprivation, $P H Q-9$ Patient Health Questionnaire-9 item scale, PSEFSM Perceived Self-efficacy for Fatigue Self-management, $P W I$ Personal Wellbeing Index, $Q o L$ quality of life, $R C T$ randomised controlled trial, SPIRIT Standard Protocol Items: Recommendations for Interventional Trials

Acknowledgments The study was funded by Macmillan Cancer Support as part of the Macmillan Survivorship Research Group programme. We thank the participants and clinicians and their teams who contributed to this study. We also thank members of the Macmillan Survivorship Research Group who supported the study and members of the Trial Steering Committee.

\section{Compliance with ethical standards}

Informed consent Informed consent was obtained from all individual participants included in the study.

Conflict of interest The authors declare that they have no competing interests.

Open Access This article is distributed under the terms of the Creative Commons Attribution-NonCommercial 4.0 International License (http:// creativecommons.org/licenses/by-nc/4.0/), which permits any noncommercial use, distribution, and reproduction in any medium, provided you give appropriate credit to the original author(s) and the source, provide a link to the Creative Commons license, and indicate if changes were made.

\section{References}

1. World Cancer Research Fund/American Institute for Cancer Research (2007) Food, nutrition, physical activity, and the prevention of cancer: a global perspective. AICR, Washington DC 
2. Foster $\mathrm{C}$ et al (2009) Psychosocial implications of living 5 years or more following a cancer diagnosis: a systematic review of the research evidence. Eur J Cancer Care (Engl) 18(3):223-247

3. Richards M, Corner J, Maher J (2011) The National Cancer Survivorship Initiative: new and emerging evidence on the ongoing needs of cancer survivors. Br J Cancer 105(Suppl 1):S1-S4

4. Richardson A (1995) Fatigue in cancer patients: a review of the literature. Eur J Cancer Care 4:20-32

5. Brown LF, Kroenke K (2009) Cancer-related fatigue and its associations with depression and anxiety: a systematic review. Psychosomatics 50(5):440-447

6. Fossa S, Dahl A, Loge J (2003) Fatigue, anxiety, and depression in long-term survivors of testicular cancer. J Clin Oncol 21:1249-1254

7. Minton $\mathrm{O}$ et al (2008) A systematic review and meta-analysis of the pharmacological treatment of cancer-related fatigue. J Natl Cancer Inst 100:1155-1166

8. Cramp, F. and J. Byron-Daniel (2012) Exercise for the management of cancer-related fatigue in adults. Cochrane Database Syst Rev

9. Reif K, de Vries U, Petermann F (2012) What does really help against cancer-related fatigue? An overview of systematic reviews. Pflege 25(6):439-457

10. Buhrman $\mathrm{M}$ et al (2004) Controlled trial of internet-based treatment with telephone support for chronic back pain. Pain 111:368-377

11. United States Census Bureau (2013) Computer and Internet use in the United States, File, T and Ryan, C., in American Community Survey Reports

12. Office for National Statistics (2013) ONS-Internet access quarterly update, Q4

13. Foster C, Fenlon D (2011) Recovery and self-management support following primary cancer treatment. Br J Cancer 105(Suppl 1):S21S28

14. Bandura A (1997) Self efficacy: the exercise of control. WH Freeman and Company, New York

15. Foster $\mathrm{C}$ et al (2015) Cancer survivors' self-efficacy to self-manage in the year following primary treatment. J Cancer Surviv 9(1):11-19

16. Yun YH et al (2012) Web-based tailored education program for disease-free cancer survivors with cancer-related fatigue: a randomized controlled trial. J Clin Oncol 30(12):1296-1303

17. Bantum EO et al (2014) Surviving and thriving with cancer using a Web-based health behavior change intervention: randomized controlled trial. J Med Int Res 16(2), e54

18. Lorig K et al (2001) Chronic disease self-management program2-year health status and health care utilization outcomes. Med Care 39:1217-1223

19. Lev EL et al (2001) An intervention to increase quality of life and self-care self-efficacy and decrease symptoms in breast cancer patients. Sch Inq Nurs Pract 15(3):277-294

20. Moher D et al (2012) CONSORT 2010 explanation and elaboration: updated guidelines for reporting parallel group randomised trials. Int J Surg 10:28-55

21. Chan A et al (2013) SPIRIT 2013 explanation and elaboration: guidance for protocols of clinical trials. BMJ 346:e7586

22. Hare J et al (2009) LifeGuide: a platform for performing web-based behavioural interventions. WebSci'09: Society On-Line, Athens, Greece
23. Foster, C., et al (2015) Managing fatigue after cancer treatment: development of RESTORE, a web-based resource to support selfmanagement. Psychooncology. (DOI: 10.1002/pon.3747)

24. Macmillan Cancer Support (2011) Coping with fatigue leaflet. Macmillan Cancer Support, London

25. Grimmett $\mathrm{C}$ et al (2013) RESTORE: an exploratory trial of an online intervention to enhance self-efficacy to manage problems associated with cancer-related fatigue following primary cancer treatment: study protocol for a randomized controlled trial. Trials 14(1):184

26. National Comprehensive Cancer Network (2013) NCCN clinical practice guidelines in oncology - cancer-related fatigue. Version 1. Fort Washington PA

27. Hoffman A et al (2011) The development and testing of an instrument for perceived self-efficacy for fatigue self-management. Cancer Nurs 34:167-175

28. Foster $\mathrm{C}$ et al (2013) Developing a scale to measure self-efficacy to SELF-manage problems following cancer treatment. Psychooncology 22(16-16):16

29. Lorig KR et al (2001) Effect of a self-management program on patients with chronic disease. Eff Clin Pract 4(6):256-262

30. Cella D et al (1993) The functional assessment of cancer therapy scale: development and validation of the general measure. J Clin Oncol 11:570-579

31. Cummins R et al (2003) Developing a national index of subjective wellbeing: the Australian unity wellbeing index. Soc Indic Res 64: 159-190

32. Kroenke K, Spitzer R, Williams J (2001) The PHQ-9: validity of a brief depression severity measure. J Gen Intern Med 16:606-613

33. Mendoza T et al (1999) The rapid assessment of fatigue severity in cancer patients - use of the brief fatigue inventory. Cancer 85 : 1186-1196

34. Myall M, May CR, Grimmett C, May CM, Calman L, Richardson A, Foster CL (2015) RESTORE: an exploratory trial of a webbased intervention to enhance self-management of cancer-related fatigue: findings from a qualitative process evaluation. BMC Med Inform Decis Mak 15:94

35. Armes J et al (2007) A randomized controlled trial to evaluate the effectiveness of a brief, behaviorally oriented intervention for cancer-related fatigue. Cancer 110(6):1385-1395

36. Bower J et al (2000) Fatigue in breast cancer survivors: occurrence, correlates, and impact on quality of life. J Clin Oncol 18:743

37. Kralik D et al (2004) Chronic illness self-management: taking action to create order. J Clin Nurs 13(2):259-267

38. Department of Culture (2009) Digital Britain: final report

39. Martin F et al (2013) Development and qualitative evaluation of a self-management workshop for testicular cancer survivor-initiated follow-up. Oncol Nurs Forum 40(1):E14-E23

40. Peels DA et al (2012) Differences in reach and attrition between Web-based and print-delivered tailored interventions among adults over 50 years of age: clustered randomized trial. J Med Int Res 14(6):146-158

41. Christensen H, Griffiths KM, Farrer L (2009) Adherence in internet interventions for anxiety and depression: systematic review. J Med Internet Res 11(2):e13.45

42. Bennett GG, Glasgow RE (2009) The delivery of public health interventions via the Internet: actualizing their potential. Annu Rev Public Health 30:273-292 